Stewart A, Brice P, Burton H, Pharoah P, Sanderson S, Zimmern R

Genetics, Health Care and Public Policy:

An Introduction to Public Health Genetics

Cambridge, Cambridge University Press, 2007

ISBN 0521529077

DOI: $10.1159 / 000106566$

\section{A New Community Genetics and Public Health Genomics Textbook}

Teaching community genetics or public health genetics to medical students or students in health sciences has not always been easy. One of the difficulties has been the fact that the main textbooks available require an understanding of English, genetics as well as public health far above the average MSc level in Europe. The new introductory textbook Genetics, Health Care and Public Policy brings together basic concepts in genetics and molecular science, definitions and history, genetic technology, epidemiology, genetic medicine, genetics in health services, ethical, legal and social implications in a volume of only 300 pages. In a rapidly developing and multidisciplinary field an update on available techniques and current debates is also welcome. The journal Community Genetics (established in 1998) and its Editor-in-Chief Leo ten Kate are discussed in a paragraph where community genetics is defined as a subdiscipline of clinical genetics.

In the book molecular genetics is dealt with on a basic level, enabling policy makers as well as students to improve their knowledge that might be relevant for their daily practice. DNA makes RNA makes protein. Not only the nucleus contains DNA, but DNA mutations in mitochondria may lead to disorders with maternal inheritance. Epigenetic mechanisms are briefly explained. Cancer is a genetic disease, where mutations accumulate: not only somatic alterations but also unrepaired errors in DNA replication.

An introduction to public health genetics, genetic epidemiology, genetics and medicine, genetics in health services, ethical, legal and social implications of genetics and policy implications are the topics of the other chapters. Delicate topics are dealt with in an open and clear way. Does the current availability of antenatal testing, accompanied by the option of abortion if the fetus proves to be affected, constitute eugenetics? The argument is that the availability of genetic testing and selective abortion is morally acceptable as long as the reason is to allow individuals to exercise reproductive choice, and state coercion is not involved.

\section{Comments}

This book is an important step in teaching community genetics and public health genomics to a wider audience. Complicated and delicate topics are discussed in a relatively easily accessible way, and there is an explanation of basic concepts from a diversity of fields (molecular genetics, epidemiology), a reflection of current debates and advice for further reading. The limitation of this book differs from previous American books: this time the focus is on the UK and not on the USA. Teaching modules still have to develop their own (inter)national scope. The legal and regulatory framework as well as the organization of health care and the list of key 'think tank' reports in each country are different. The oversights in this book will certainly stimulate non-UK readers to make their own analysis of the interface between public health and genomics in their countries.

M.C. Cornel, Amsterdam

\begin{abstract}
Parsons EP, King JT, Israel JA, Bradley DM
Mothers' Accounts of Screening Newborn Babies in Wales (UK)
\end{abstract}

Midwifery 2007;23:59-65

\section{DOI: $10.1159 / 000106567$}

\section{Informed Consent for Newborn Screening?}

The uptake of newborn screening has been largely taken as a given, and there has been little recognition that because it is genetic screening it should therefore require more rigorous consent. One explanation could be the long-standing acceptance that newborn screening is concerned with treatable diseases such as phenylketonuria (PKU) and congenital hypothyroidism $(\mathrm{CHT})$, and is therefore in the domain of public health medicine. Reported in this paper are the findings of a qualitative study, carried out in Wales, UK, to explore mothers' experiences of newborn baby screening. Wales is unique in the UK because it offers screening for both treatable and untreatable diseases. The four diseases screened for are PKU, CHT, cystic fibrosis, and for boys only, Duchenne muscular dystrophy (DMD). DMD is an opt-in test, and mothers are asked to sign the screening card to indicate their consent. No signature is required for the other three tests.

Midwifes were asked to identify a sequential group of 25-30 mothers who fulfilled the following criteria: had a vaginal birth of a singleton live baby who did not have to be admitted to a neonatal unit; maternal records included details of GP, health visitor and midwife; mother was English speaking, and the baby's newborn tests were normal. When the babies were $4-6$ weeks old, the researcher contacted by letter those mothers who had agreed to join the study. Semistructured interviews took place 6-9 weeks after birth, lasting 30-40 min. All interviews were carried out by one of the authors, a midwife, and consisted of the following main topics: their experience of pregnancy, birth, their first days at home, and newborn screening.

Twenty-five mothers were approached and 18 agreed to be interviewed. All the mothers had accepted screening for PKU, CHT and cystic fibrosis, and all 10 mothers with baby boys had agreed to the DMD test. Sixteen were first-time mothers.

In Wales, mothers obtain information about newborn screening from an information leaflet and talking to their midwife. Three mothers reported that newborn screening had been mentioned during their pregnancy but only one had received a leaflet. The remaining mothers said that they had only been given infor- 
mation after their baby's birth. They found it difficult to absorb new detailed information and to cope with the newborn baby at the same time. Most mothers were dissatisfied concerning the information process for the following three main reasons: a lack of information, literature overload, and the midwife not spending enough time discussing it. The newborn baby screening was not perceived as different from other tests, and was portrayed as a relatively unimportant routine procedure. Although screening was seen as a routine test, it was described as an imperative: 'they should be done'.

The majority of the mothers talked about consent as being important, and there was recognition that, in practice, the consent process had been largely compromised. The high level of trust in their midwife was combined with a belief that the test was in the best interest of their baby. In contrast, mothers were quite clear that antenatal screening was not a routine test. It had been presented to them in terms of choice and a decision that they could make without any feelings of obligation or institutional expectations.

The present study showed that mothers define newborn screening as a routine procedure for a 'package of diseases'. Whatever the explanation, it is clear that mothers do not give informed consent, which they should.

\section{Comments}

Technological developments have led to an increased number of genetic tests for both treatable and untreatable diseases. Arguments in favor of screening all newborn babies for treatable diseases, such as PKU and CHT, are overwhelming. Screening for this kind of diseases is in the child's best interest, and should always be carried out. With regard to untreatable childhood onset diseases it has been argued that including these in the neonatal screening program would be of great interest for parents of an already affected child who are planning to have more children in order to prevent the birth of another affected child within this family. Further, a long medical route, filled with insecurity, can be avoided if the child is diagnosed shortly after birth. On the other hand, others argue that parents would be worried before the child actually falls ill, and these parents can therefore never happily enjoy their child.

Therefore the authors of the paper argue that the alternative would be to make a distinction between treatable and untreatable diseases, and seek different levels of consent. Neonatal screening for treatable diseases should be regarded as a routine test, since it is in the child's best interest to diagnose them as early as possible, whereas screening for untreatable (childhood onset) diseases should be presented analogue to the way in which antenatal screening is: as a decision that can be made without any feelings of obligation or institutional expectations.

A.M.C. Plass, Amsterdam 